NASA Technical Memorandum 102316

AIAA-89-2696

\title{
Large-Scale Advanced Propeller Blade Pressure Distributions: Prediction and Data
}

M. Nallasamy, O. Yamamoto, and S. Warsi

Sverdrup Technology, Inc.

NASA Lewis Research Center Group

Cleveland, Ohio

and

L.J. Bober

National Aeronautics and Space Administration

Lewis Research Center

Cleveland, Ohio

Prepared for the

25th Joint Propulsion Conference

cosponsored by the AIAA, ASME, SAE, and ASEE

Monterey, California, July 10-12, 1989 


\title{
LARGE-SCALE ADVANCED PROPELLER BLADE PRESSURE DISTRIBUTIONS: PREDICTION AND DATA
}

\author{
M. Nallasamy, O. Yamamoto, and S. Warsi \\ Sverdrup Technology, Inc. \\ NASA Lewis Research Center Group \\ Cleveland, Ohio 44135 \\ and \\ L.J. Bober \\ National Aeronautics and Space Administration \\ Lewis Research Center \\ Cleveland, Ohio 44135
}

\section{Summary}

Two Euler analysis techniques-finite difference and finite volume-were employed to predict the blade surface pressure distributions of a large-scale advanced propeller. The predicted pressure distributions were compared with wind tunnel data. Both techniques produced blade pressure distributions that are in fairly good agreement with the data over the range of test Mach numbers of 0.2 to 0.78 . However, the numerical simulations fail to predict correctly the measured pressure distributions for the low Mach number, high-power case. The data indicate the presence of a leading-edge vortex for this case. A discussion of the compressibility effects is also presented.

\section{Introduction}

Advanced propellers employ highly loaded, highly swept, thin blades with variable pitch to achieve a higher propulsive efficiency than is achieved by the current high-bypass turbofans. Model tests have been carried out in wind tunnels and in flight to understand the aerodynamics and acoustics of the advanced designs. The propeller blade flows are complicated and involve a wide range of flow regimes, such as (1) takeoff conditions, where leading edge and tip vortices influence the performance and noise generation; and (2) cruise conditions, where shock waves add to the complexity. An understanding of the complex advanced propeller blade flows is essential for improving the current design methodologies.

Scale-model tests have provided integrated performance data. To obtain aerodynamic blade data, a series of tests on a large-scale 9-ft single-rotation SR7L propeller were conducted in a transonic wind tunnel in Modane, France. The propfan was operated in a two-bladed configuration (fig. 1). This configuration was adopted for the tests because the drive power was insufficient for the eight-bladed propeller. The design characteristics of the SR7L propeller are given in table I.

The blade surface pressure was measured on a specially designed, pressure-tapped SR7L blade. There were 13 radial stations of pressure taps on each surface of the blade: 16 pressure taps were distributed along the blade chord at each radial station on the pressure (face) side and 20 were distributed on the suction (camber) surface. The taps were concentrated on the blade surface in the areas where large pressure gradients were expected. Each chordwise distribution was aligned approximately with the expected streamline on the blade at the design cruise condition (fig. 2). The blade surface pressures were recorded one radial station at a time, and the tests were conducted over the Mach number range of 0.03 to 0.78 . A description of the nature and extent of the large-scale advanced propeller wind tunnel tests is given by Campbell et al. (ref. 1).

The purpose of the present investigation is to evaluate the ability of the Euler analysis techniques to predict the blade 
surface pressure distributions. The three-dimensional Euler equations are solved by two different analysis techniques: (1) a finite difference technique (NASPROP) originally developed by Bober et al. (ref. 2), and (2) a finite volume technique developed by Denton (ref. 3). In this paper, the predicted chordwise pressure distributions and the integrated power coefficients are compared with the test data for the Mach number range from 0.2 to 0.78 .

\section{Computational Methods}

Two numerical codes are employed to simulate the flow field about the two-bladed single-rotation propeller (SR7L) subject to conditions prescribed in the Modane, France, test. Both codes use an asymptotic time-marching approach to obtain the steady-state solutions of the three-dimensional Euler equations governing the propeller flow. Brief descriptions of each code follow.

\section{Finite Difference Euler Solver (NASPROP)}

The finite difference Euler solver (NASPROP) was originally developed by Bober et al. (ref. 2). Their threedimensional Euler analysis was the first to provide detailed solutions of the advanced propeller flow field. It employs the numerical method of Beam and Warming. Since then the code has been rewritten incorporating improved boundary conditions and has been vectorized for the CRAY computer.

The three-dimensional, rotational, inviscid flow field about a propeller is governed by the Euler equations. The equations, in conservative differential form, are transformed from a cylindrical coordinate system to a time-dependent, body-fitted, curvilinear reference frame. The transformed equations are treated as a system of coupled first-order, autonomous differential equations. The equations are cast in nondimensional form. First-order-accurate implicit Euler time differencing is employed, and the flux vectors are linearized by Taylor series expansion up to second order in time. The spatial derivatives are replaced by central differences with appropriate one-sided differences at the boundaries. The resulting implicit operators involve inversions of a block tridiagonal system. The system can be reduced to scalar tridiagonal matrices by applying a diagonalization technique (ref. 4) to the flux Jacobian matrices. Constant coefficient, second-order implicit and fourth-order explicit dissipation terms are added for stability considerations. A periodic tridiagonal matrix inversion is employed in the circumferential direction. Further details of the solution procedure and the implementation of the boundary conditions can be found in references 5 and 6 .

\section{Finite Volume Euler Solver (Denton)}

The finite volume Euler solver was originally developed by Denton for the analysis of turbomachinery flows (ref. 3) and was later modified to compute the advanced propeller flow field (refs. 7 and 8). In Denton's analysis, the threedimensional Euler equations are written in a coordinate frame rotating with the blade. The governing equations are solved in the physical domain on a grid that is generated algebraically. The flow domain is discretized by using three kinds of surfaces: the bladewise surface, the streamwise surface, and the spanwise surface. The computational nodes are located at the corners of each volume element. The fluxes of mass, momenta, and energy through each face are calculated by using the averages of flow properties stored at the corners of that face.

Denton's explicit time-marching method employs an opposed difference scheme. The scheme uses upwind differences for fluxes of mass and momenta, and downwind differences for pressure in the streamwise direction. The derivatives in the circumferential direction are evaluated by using central differences. The time-marching method uses a time step such that the Courant number is close to unity. The method itself ensures stability by solving the flow equations in the order of continuity, energy, and momentum. A variable time step appropriate for each volume element is used to accelerate convergence. The method is of first-order accuracy and is modified to achieve greater accuracy by adding a lagged correction factor to correct the downwinded pressure. A smoothing factor is used after each time step to smooth out any waviness in the circumferential direction. The convergence is judged on the basis of mass conservation and the maximum change in the axial velocity component. Further details of the solution procedure and the implementation of the boundary conditions can be found in references 3 and 8 .

\section{Computational Grid}

In this paper, the steady-state propeller flow field with the propeller axis at zero angle of attack is computed. Hence only one blade passage needs to be considered as a computational domain since the flow field is periodic in the blade-to-blade direction.

The computational domain is discretized by three types of surfaces. The bladewise surfaces are bounded by two planesone plane containing the suction side of one blade and the other plane containing the pressure side of the adjacent blade (fig. 3(a)). The surfaces are clustered near the blades, and larger spacings are used between the blades. The streamwise surfaces (fig. 3(b)) are placed in the radial direction and are limited by the spinner-nacelle surface and the far field boundary. The far field boundary is placed at two blade radii beyond the blade tip. Small spacings are used in the blade tip region to capture the tip vortex flow. The spanwise surfaces are bounded by inlet and exit planes in the axial direction (fig. 3(c)). The inlet plane is placed at one radius upstream of the spinner, and the exit plane is located at three radii behind the blade. Grid points are clustered near the leading and trailing edges. 
From a few grid-sensitivity calculations, 35 chordwise and 20 spanwise points on each side of the blade are found to be adequate to obtain a solution that is nearly independent of the grid. Figure 3(d) shows the distribution of grid points on the blade surface. A total of 61 by 33 by 35 (axial, radial, and circumferential) grid points is used to discretize the whole computational space. The same grid density on the blade surfaces, and similar grid arrangements, are employed by the two computer programs.

\section{Results and Discussion}

The steady three-dimensional flow field of an advanced propeller, SR7L, is considered here. The Euler equations governing the flow field are solved by a finite difference and a finite volume technique. The numerical results are compared with wind tunnel data.

The numerical data for nine of the Modane test cases (table II) were generated by the two numerical codes independently. The data input to the codes were the same. In particular, exactly the same blade setting angles were used in both codes. Although each code used its own grid generator for high Mach number runs, both used the same grid generator for low Mach number $(0.2)$ runs. The results are compared with the wind tunnel data in the form of surface pressure contours, chordwise pressure distributions, and total power coefficients.

The pressure coefficient is defined in this paper as

$$
C p=\sum_{i j} \frac{P_{i j}-P_{\infty}}{0.5 \rho_{\infty}\left(V_{\infty}^{2}+r_{i j}^{2} \omega^{2}\right)}
$$

where $P_{i j}$ and $r_{i j}$ are the local pressure and the radial distance to the data point; $P_{\infty}, \rho_{\infty}$, and $V_{\infty}$ are the pressure, density, and velocity of the free stream; and $\omega$ is the rotational speed $(\mathrm{rad} / \mathrm{sec})$ of the blade. The computed pressure is interpolated to match the radial location of the pressure taps. The total power coefficient of the propeller is computed from the blade surface pressure by

$$
C P=\sum_{i j} \frac{2 \pi}{\rho_{\infty} \Omega^{2} D^{5}} r_{i j}\left(P_{i j} \Delta \overrightarrow{\mathrm{A}}_{i j}\right)_{\ominus} B
$$

where $\Delta \vec{A}_{i j}$ is the elemental surface area formed by four neighboring grid points, $r_{i j}$ is the radial distance of the element, $D$ is the diameter of the propeller, $\Omega$ is the rotational velocity (rev/sec), and $B$ is the number of blades. The subscript $\theta$ denotes the component in the tangential direction.

The Modane test results (ref. 9) and the computed power coefficients are presented in table II. The Mach number and the advance ratio for the computations were matched with the experimental conditions. The use of nominal blade setting angles for computations resulted in power coefficients higher than the measured ones. Based on the computed power coefficients, a second set of blade setting angles were chosen within the tolerance limits, to make the predictions closer to the data. These blade setting angles, which were used in the computations, and the resulting power coefficients are given in the table. For high Mach number tests, the power coefficients are still overpredicted. The blade setting angles may have to be lowered beyond the tolerance limits to improve the agreement in these cases. No further change in the blade setting angle was made.

The power coefficients predicted by the numerical codes closely follow the power trends that are observed in the experiments. Tests 5 and 6 are designed to compare the power at cutback and takeoff conditions at low Mach number. The predictions show the correct trend, except that the NASPROP code underpredicts the measured value in test 6 .

Tests 7, 8, and 9 show the power trend when the blade angle is changed while the Mach number and the advance ratio are kept constant. For low-power range, NASPROP results show excellent agreement with the wind tunnel data.

Tests 10 through 12 are designed to show the Mach number effect when the blade angle is kept constant. Through these tests the power stays relatively constant, although the results of the finite difference code show a slight increase with Mach number. The Denton code results show nearly the same trend as the data. The last test was conducted as a variation of test 12 with a different advance ratio. The inverse relation between the power coefficient and the advance ratio is correctly predicted by both numerical codes; however, the finite difference code tends to overpredict the power consistently for these cases.

Three representative test cases were chosen for detailed comparisons: test 12 for a high Mach number (0.775), test 8 for an intermediate Mach number (0.5), and test 5 for a low Mach number (takeoff, 0.2).

Contours of measured surface pressure for test 12 are shown in figure 4 . Because of the coarseness of the experimental data points, the blade shape and the contour lines are not smoothly constructed. Although there are 20 pressure taps in each of the 13 radial stations on the suction side and 16 taps on each pressure surface radial station, data were recorded for only 10 radial stations for test 12 . Also, a few pressures were not valid at several radial stations. Missing data were approximated by interpolation or extrapolation from neighboring data.

The corresponding data generated by the two numerical codes are also shown in figure 4 . Similarity between the numerical results is clearly seen. On the suction side (fig. 4(a)), the contour features can be easily identified with those of the data. The agreement of the predictions with the data on the pressure surface (fig. 4(b)) is not as good as that on the suction surface.

Detailed chordwise pressure distributions at four radial stations $(r / R=0.569,0.739,0.862$, and 0.975$)$ are shown in figure 5(a), (b), (c), and (d), respectively. Better agreement 
is seen on the suction side, except that a strong shock is generated at the outboard trailing edge by the NASPROP code.

Although some test data are missing in this region and it is difficult to assess exact flow, a shock as strong as that predicted by NASPROP may not have developed during the test. Contrary to the NASPROP results, the Denton code does not show the formation of a strong shock though it clearly shows the compressibility effect in the region and agrees well with the measurements. Both codes tend to overpredict the pressures on the pressure side, which is reflected in the power coefficient comparison in table II. The inverted loading near the leading-edge region obtained in the data is predicted poorly on the inboard region. This may be the result of the leadingedge geometry represented by the numerical codes not exactly matching the physical blade geometry. The estimated structural deflections at the design point cruise condition were used for all of the runs.

The surface pressures produced by the NASPROP code are compared with the test 8 data in figure 6 , and the local chordwise pressure distributions at the four radial stations are compared with test 8 data in figure 7. As in the previous case, good agreement is shown on the suction side. Except on the blade edges, the contour levels on the pressure side compare reasonably well. It should be noted that the blade outline in the test data is based on the pressure tap locations and does not represent the true outline of the blade. In general, better agreements are seen in the outboard section of the blade, and discrepancies are observed at the inboard leading-edge section.

Chordwise pressure profiles (fig. 7) reveal that the loading is fairly evenly distributed from the hub to the tip at this condition. Both codes show reasonably good agreement at all four radial stations. The pressure side is predicted better by the Denton code than by the NASPROP code.

Numerical results are compared with experimental test 5 data (Mach 0.2) in figures 8 and 9. The agreement of the predicted pressure contours with data (fig. 8) is good except for a few isolated places like the edges of the blade and the hub region. Figure 9 shows that both numerical predictions agree very well with test data, especially in the outboard section of the blade. The Denton code slightly overpredicted the pressure loading throughout the blade, which resulted in a power coefficient that was higher than the experimental value (table II).

Figure 10 shows a low Mach number $(0.2)$ case with a higher power (test 6), and presents an interesting flow phenomenon. The numerical results are represented by the Denton calculations. The code is able to capture the high-pressure loading at the leading edge reasonably well. The wind tunnel data show broadening of the suction peak on the suction side at radial stations $r / R=0.862$ and $r / R=0.905$. This behavior of the pressure curve is considered to be the result of the formation of a leading-edge vortex. Oil flow studies of surface streamlines indicate the formation of such leading-edge vortices (refs. 10 and 11). Unfortunately, with the conditions prescribed in the Modane test and the grid distribution employed in the present numerical solutions, the vortex flow has not been captured by either numerical code. Grid refinement and improved dissipation models may be necessary to predict the leading-edge vortex. Numerical solutions of the Euler equations have been found to predict the leading-edge vortices on swept wings fairly well (ref. 12).

Lastly, the Mach number effect is demonstrated in figures 11 and 12. Figure 11(a) shows the NASPROP pressure contours on the suction side for four Mach numbers, $0.5,0.6,0.7$, and 0.775 (tests $8,10,11$, and 12 , respectively) and the corresponding experimental data. The pressure surface predictions are compared with data in figure 11(b). It should be noted that for this compressibility study the blade angle is kept constant. However, for the experimental results, a slightly different blade angle was used for the Mach 0.5 case (see table II). With increasing Mach number, more significant changes in the flow occur on the suction side than on the pressure side. The predicted flow features compare well with the experimental data.

Figure 12 shows the local pressure distribution at two outboard stations, $r / R=0.806$ and 0.939 , for the compressibility study. Although no significant changes are observed for low Mach numbers, the evidence of a compression wave starts to develop on the suction side when the Mach number reaches about 0.7. At a free-stream Mach number of 0.78 , the compression wave fully develops to a trailing-edge shock. The NASPROP results agree with the experimental data up to the development of the compression wave. However, the strong trailing-edge shock formation is not indicated by the test data or the results of the Denton code. The reasons for this discrepancy are not clear.

\section{Concluding Remarks}

The detailed chordwise pressure distributions of an advanced propeller were predicted by two numerical techniques of solving the three-dimensional Euler equations-finite difference and finite volume-and were compared with wind tunnel data. In general, both techniques predicted the blade surface pressure distributions fairly well for the range of Mach numbers considered ( 0.2 to 0.78$)$. The strong trailing-edge shock predicted by the finite difference code (NASPROP) at high Mach numbers $(0.775$ and 0.78$)$ was not observed either in the experimental data or in the Denton code predictions. In the intermediate Mach number range $(0.5$ to 0.7$)$ the agreement of the predictions with data was good. Generally, the predictions showed better agreement at the outboard section than at the inboard section. For the low Mach number (0.2) cases, the agreement of the predictions with data was best for the low-power case. The data for the high-power case seems to show the formation of a leading-edge vortex that is not captured by the numerical techniques. Grid refinement and improved dissipation models may be required to capture the leading-edge vortex. 


\section{References}

1. Campbell, W.A.; Wainauski, H.S.; and Bushnell, P.R.: A Report on High Speed Wind Tunnel Testing of the Large Scale Advanced Propfan. AIAA Paper 88-2802, 1988.

2. Bober, L.J.; Chaussee, D.S.; and Kutler, P.: Prediction of High Speed Propeller Flow Fields Using a Three-Dimensional Euler Analysis. AIAA Paper 83-0188, 1983 (NASA TM-83065).

3. Denton, J.D.: Time Marching Methods for Turbomachinery Flow Calculations. Numerical Methods in Applied Fluid Dynamics. B. Hunt, ed., Academic Press, 1980, pp. 473-493.

4. Pulliam, T.H.; and Chaussee, D.S.: A Diagonal Form of an Implicit Approximate Factorization Algorithm. J. Comput. Phys., vol. 39, no. 2, Feb. 1981, pp. 347-363.

5. Barton, J.M.; Yamamoto, O.; and Bober, L.J.: Euler Analysis of Transonic Propeller Flows. J. Propulsion Power, vol. 3, no. 3, MayJune 1987, pp. 277-282.

6. Yamamoto, O.; Barton, J.M.; and Bober, L.J.: Improved Euler Analysis of Advanced Turboprop Propeller Flows. AIAA Paper 86-1521, 1986.
7. Clark, B.J.; and Scott, J.R.: Coupled Aerodynamic and Acoustical Predictions for Turboprop. NASA-TM-87094, 1986.

8. Nallasamy, M.; Clark, B.J.; and Groeneweg, J.F.: Euler Analysis of the Three-Dimensional Flow Field of a High Speed Propeller: Boundary Condition Effects. J. Turbomachinery, vol. 109, no. 3, July 1987, pp. 332-339.

9. Bushnell, P.: Measurement of the Steady Surface Pressure Distribution on a Single Rotation Large Scale Advanced Prop-Fan Blade at Mach Numbers from 0.03-0.78. NASA-CR-182124, 1988.

10. Stefko, G.L.; Rose, G.E.; and Podboy, G.G.: Wind Tunnel Performance Results of an Aeroelastically Scaled 2/9 Model of the PTA Flight Test Prop-Fan. AIAA Paper 87-1893, 1987 (NASA TM-89917).

11. Vaczy, C.M.; and McCormick, D.C.: A Study of the Leading Edge Vortex and Tip Vortex on Propfan Blades. J. Turbomachinery, vol. 109, no. 3, July 1987 , pp. 325-331.

12. Rizzetta, D.P.; and Shang, J.S.: Numerical Simulation of Leading Edge Vortex Flows. AIAA J., vol. 24, no. 2, Feb. 1986, pp. 237-245.

TABLE I.-SR7L PROPELLER DESIGN PARAMETERS

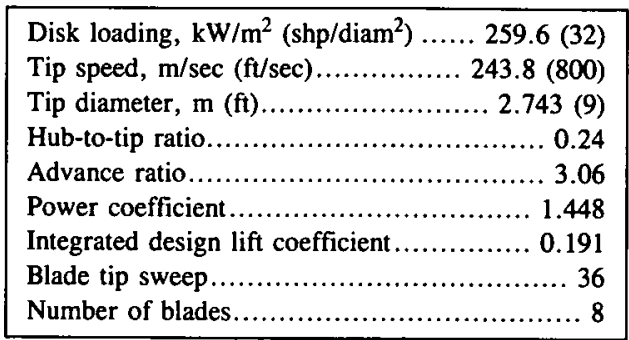

TABLE II.-MODANE TEST OPERATING CONDITIONS

\begin{tabular}{|c|c|c|c|c|c|c|c|}
\hline \multirow{3}{*}{$\begin{array}{c}\text { Test } \\
\text { number }\end{array}$} & \multirow{3}{*}{$\begin{array}{c}\text { Mach } \\
\text { number }\end{array}$} & \multirow{3}{*}{$\begin{array}{c}\text { Advance } \\
\text { ratio }\end{array}$} & \multirow{2}{*}{\multicolumn{2}{|c|}{$\begin{array}{l}\text { Blade angle, } \\
\text { deg }\end{array}$}} & \multicolumn{3}{|c|}{ Power coefficient } \\
\hline & & & & & \multirow{2}{*}{ Test } & \multirow{2}{*}{$\begin{array}{l}\text { NASPROP } \\
\text { calculation }\end{array}$} & \multirow{2}{*}{$\begin{array}{c}\text { Denton } \\
\text { calculation }\end{array}$} \\
\hline & & & Test & Calculation & & & \\
\hline 5 & 0.2 & 0.88 & $26.6 \pm 1.0$ & 25.6 & $0.100 \pm 0.002$ & 0.108 & 0.121 \\
\hline 6 & .2 & .883 & $31.3 \pm .9$ & 30.4 & $.250 \pm .001$ & .193 & .283 \\
\hline 7 & .5 & 3.083 & $58.5 \pm 1.0$ & 57.5 & $.642 \pm .009$ & .564 & .692 \\
\hline 8 & .5 & 3.067 & $55.1 \pm .8$ & 55.0 & $.361 \pm .005$ & .369 & .344 \\
\hline 9 & .5 & 3.071 & $51.8 \pm .9$ & 51.6 & $.110 \pm .004$ & .106 & .112 \\
\hline 10 & .6 & 3.078 & $54.5 \pm 1.3$ & 53.6 & $.230 \pm .006$ & .254 & .262 \\
\hline 11 & .7 & 3.064 & $54.5 \pm 1.3$ & 53.6 & $.228 \pm .006$ & .271 & .244 \\
\hline 12 & .775 & 3.088 & $54.5 \pm 1.3$ & 53.6 & $.227 \pm .003$ & .286 & .247 \\
\hline 13 & .78 & 3.209 & $54.5 \pm 1.3$ & 53.6 & $.111 \pm .005$ & .207 & .134 \\
\hline
\end{tabular}




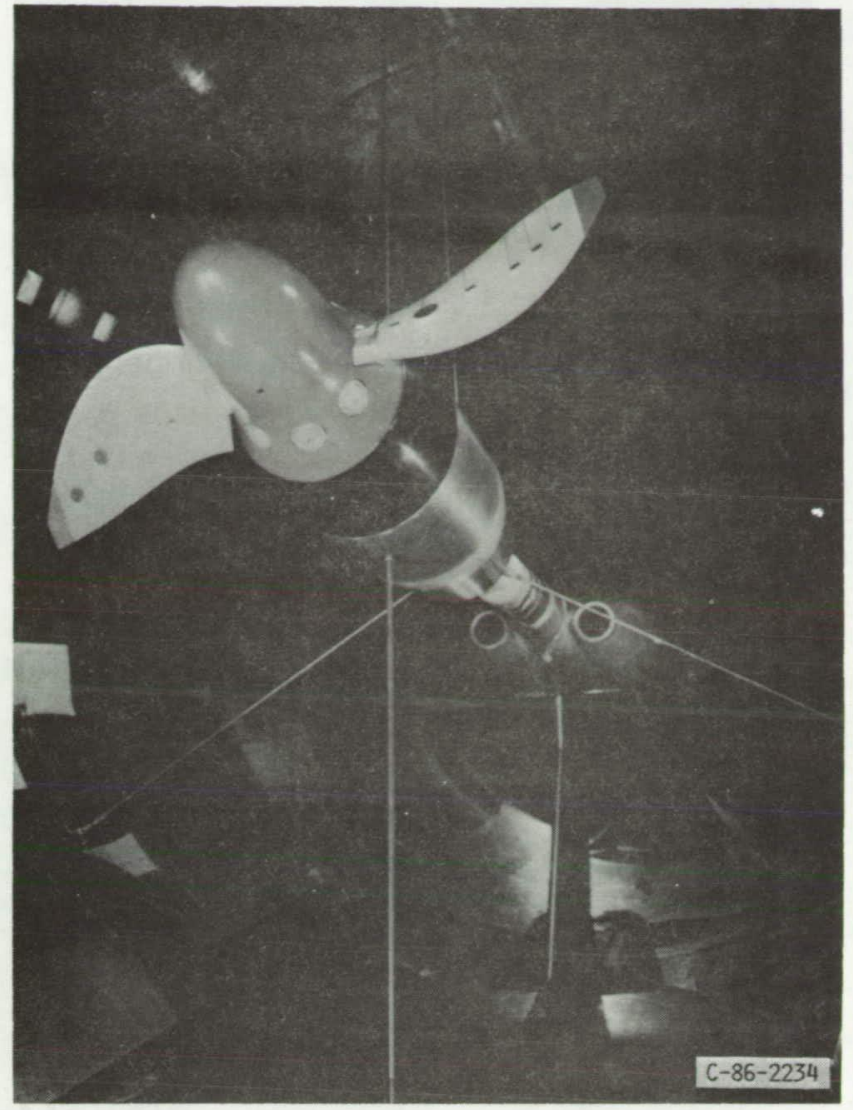

ORIGINAL PAGE IS OF POOR QUALITY

Figure 1.-SR7L propfan installed in the S1-MA transonic test section.

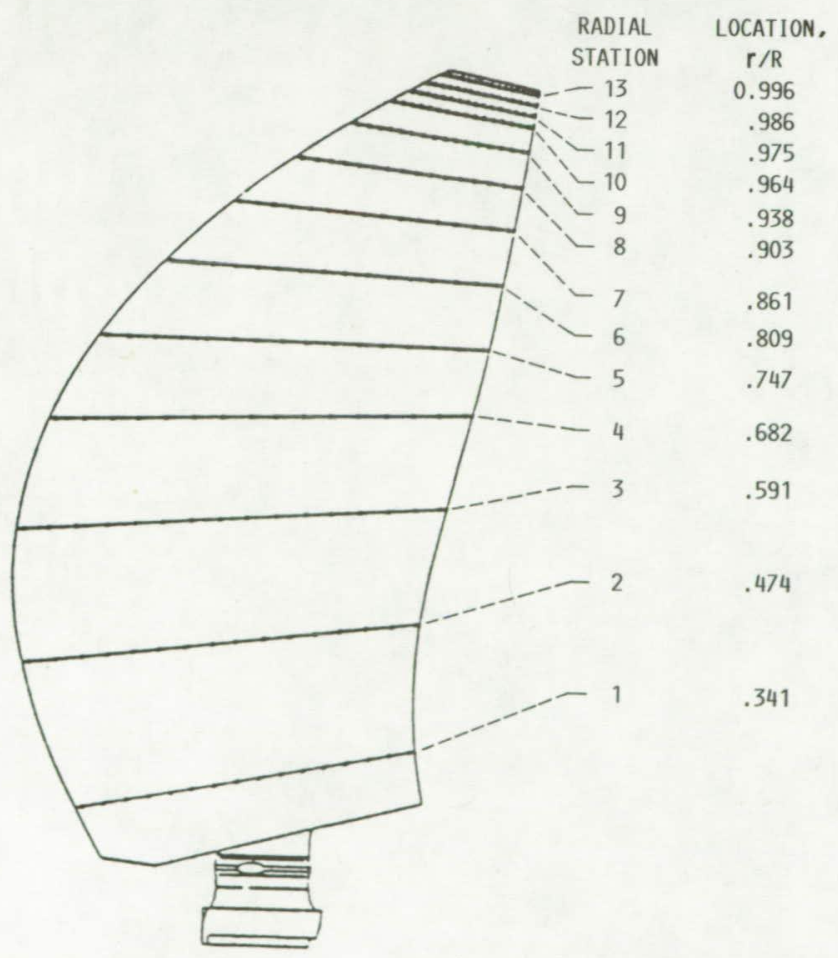

Figure 2.-Pressure tap locations. 


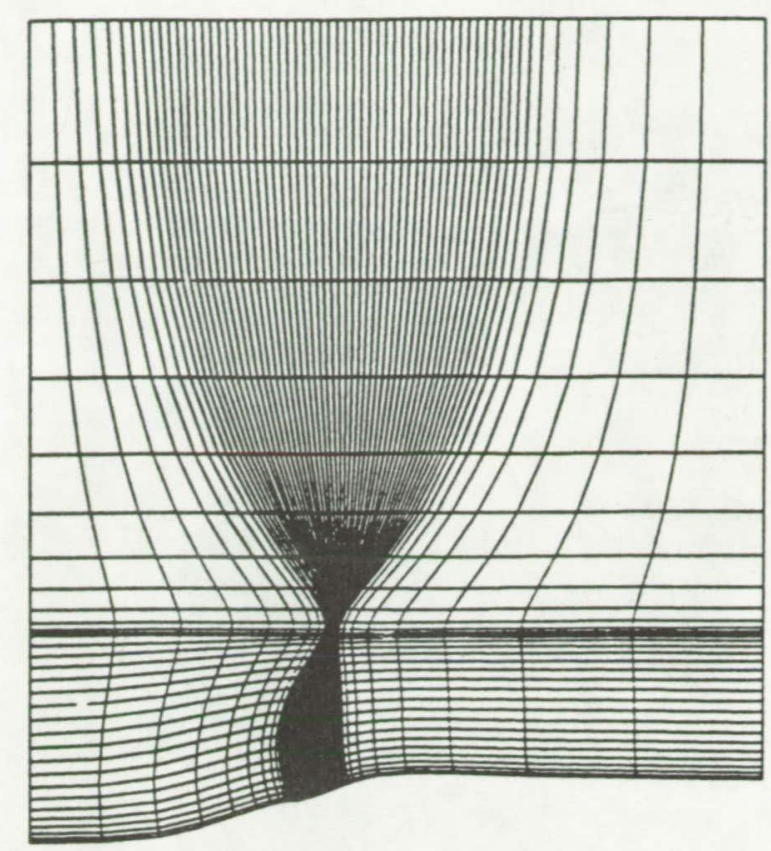

(a)

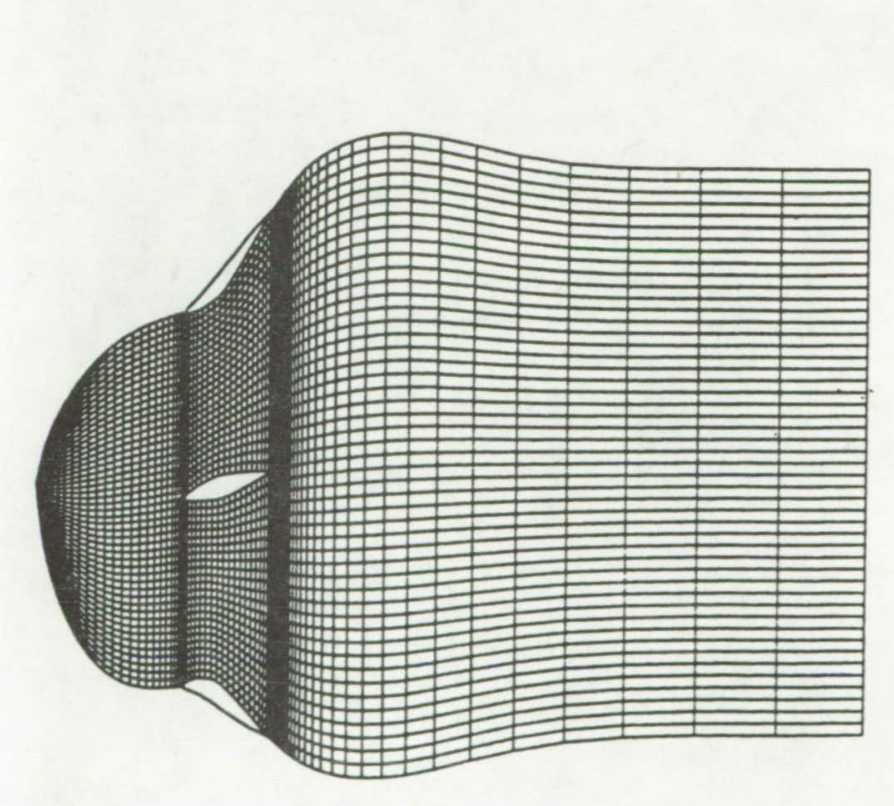

(b)

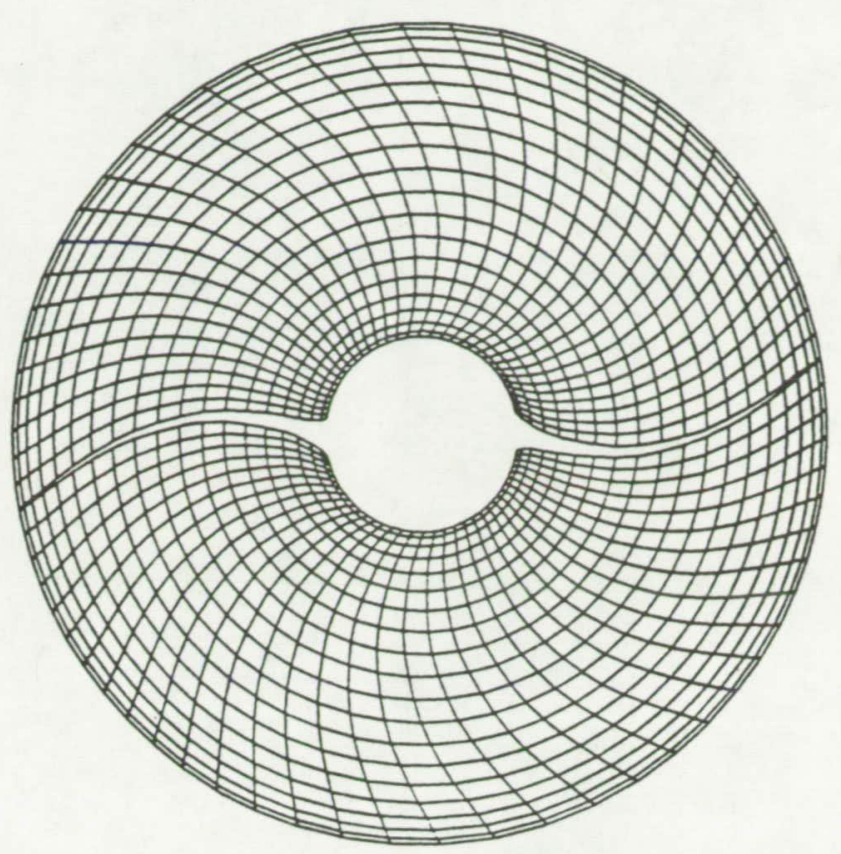

(c) (d)

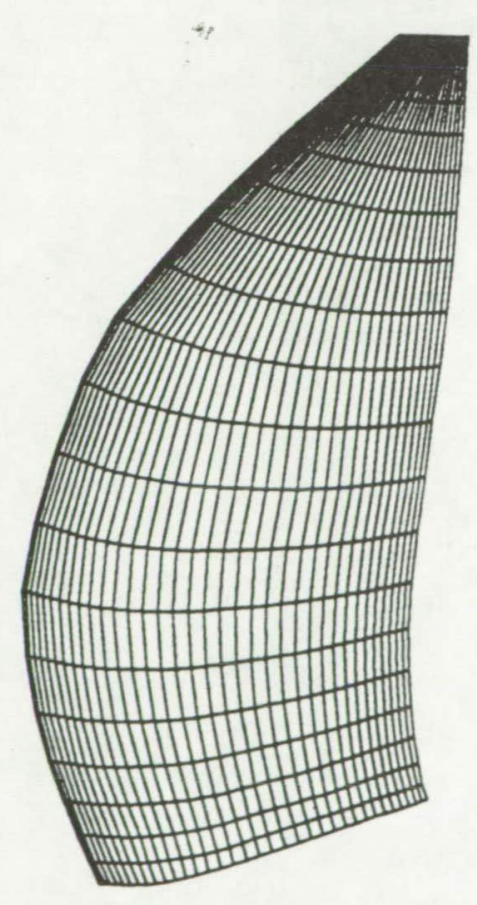

(a) Bladewise surfaces.

(b) Streamwise surfaces.

(c) Spanwise surfaces.

(d) Distribution of grid points on the blade surface.

Figure 3.-Typical computational grid. 


\section{ORIGINAL PAGE COLOR PHOTOGRAPH}

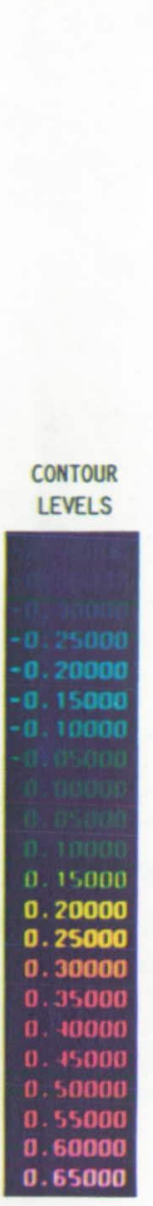

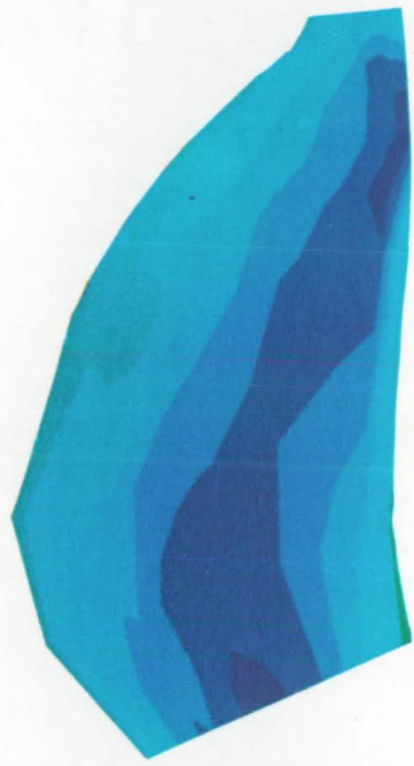

EXPERIMENTAL DATA

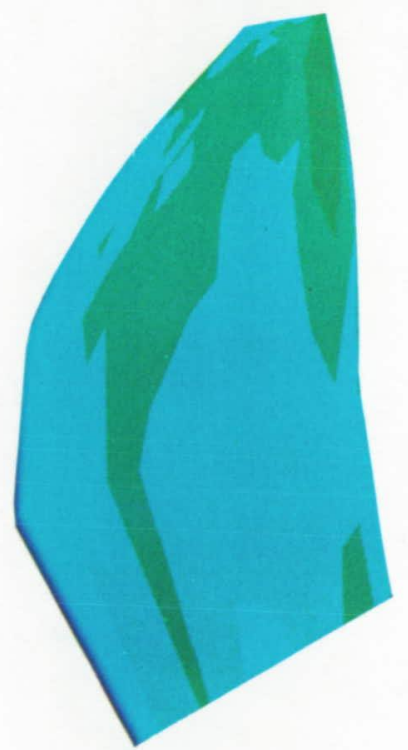

EXPERIMENTAL DATA

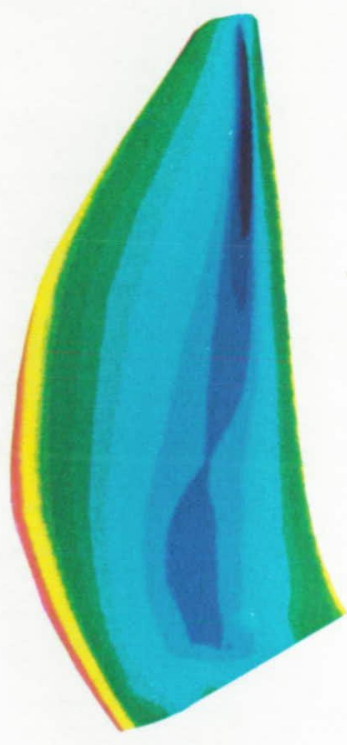

NASPROP PREDICTION

(a)

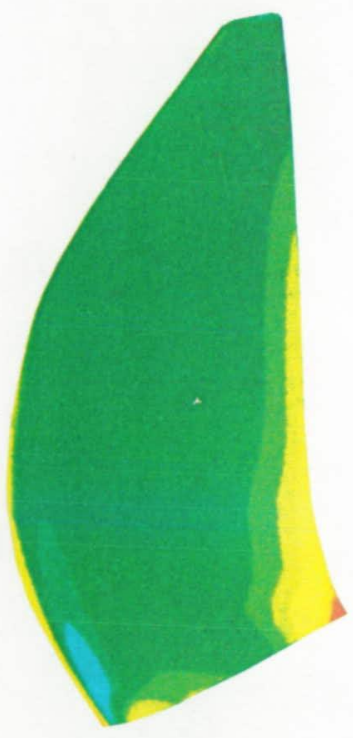

NASPROP PREDICTION

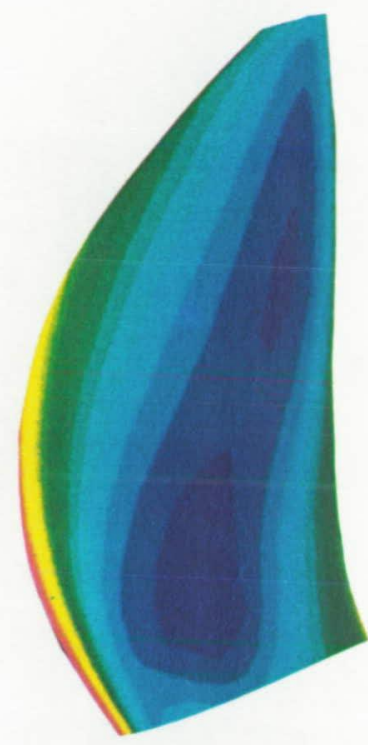

DENTON PREDICTION

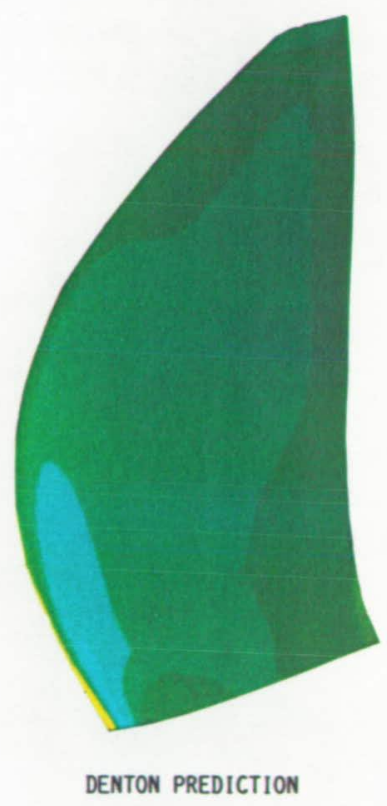

C-89-06142

(b)

(a) Suction surface.

(b) Pressure surface.

Figure 4.-Comparison of predicted and measured pressure coefficient distributions for test 12 (Mach 0.775 ). 

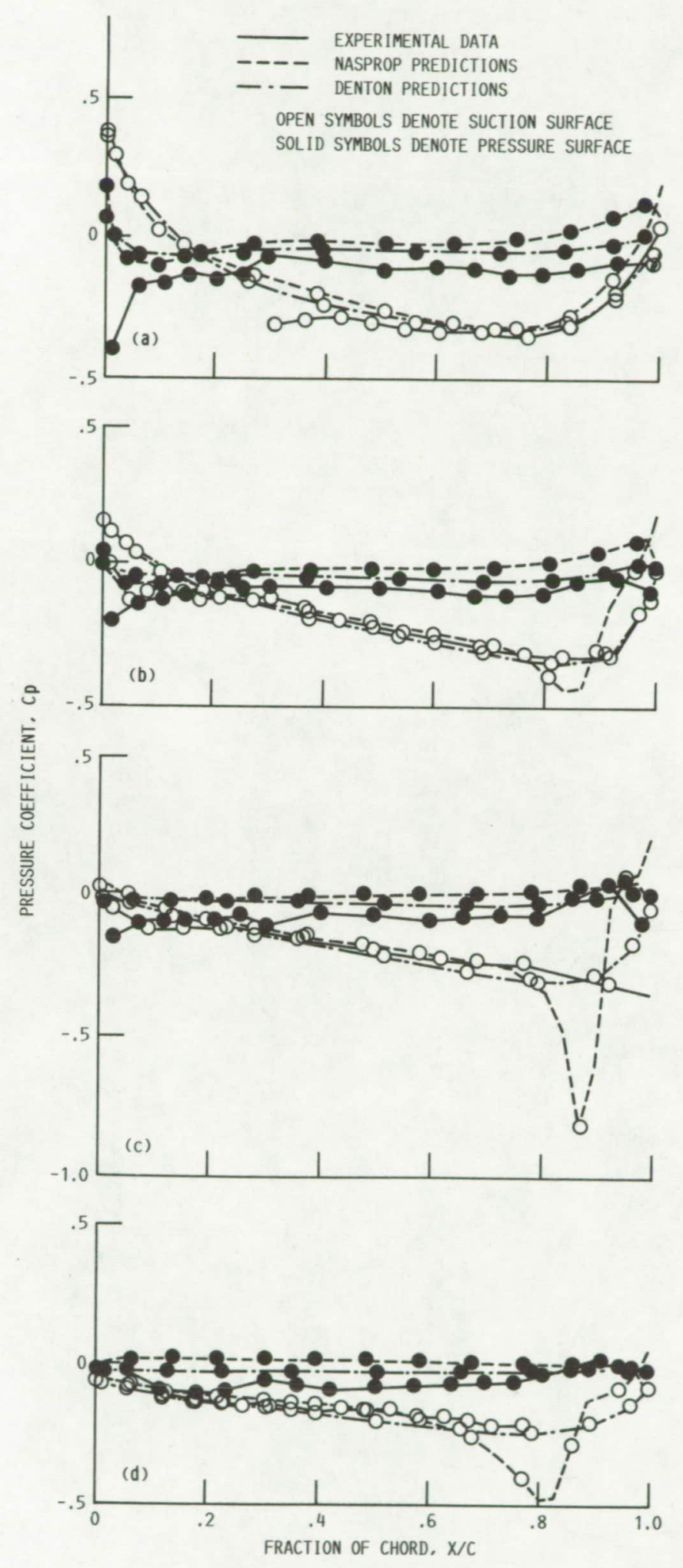

(a) Radial location, $r / R, 0.569$.

(b) Radial location, $r / R, 0.739$.

(c) Radial location, $r / R, 0.862$.

(d) Radial location, $r / R, 0.975$.

Figure 5.-Chordwise distribution of pressure coefficients for test 12 (Mach 0.775). 


\section{ORIGINAL PAGE COLOR PHOTOGRAPH}
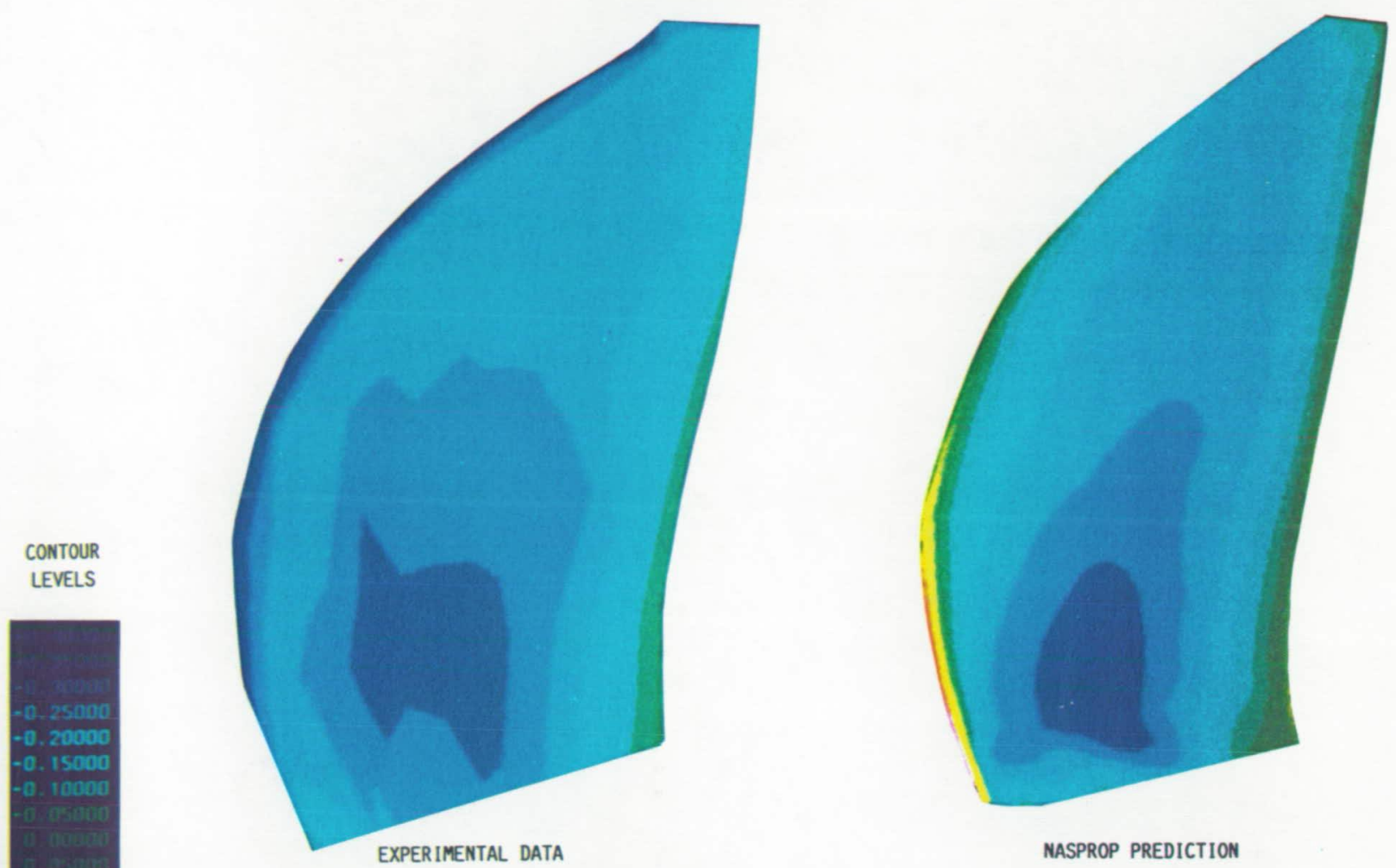

(a)
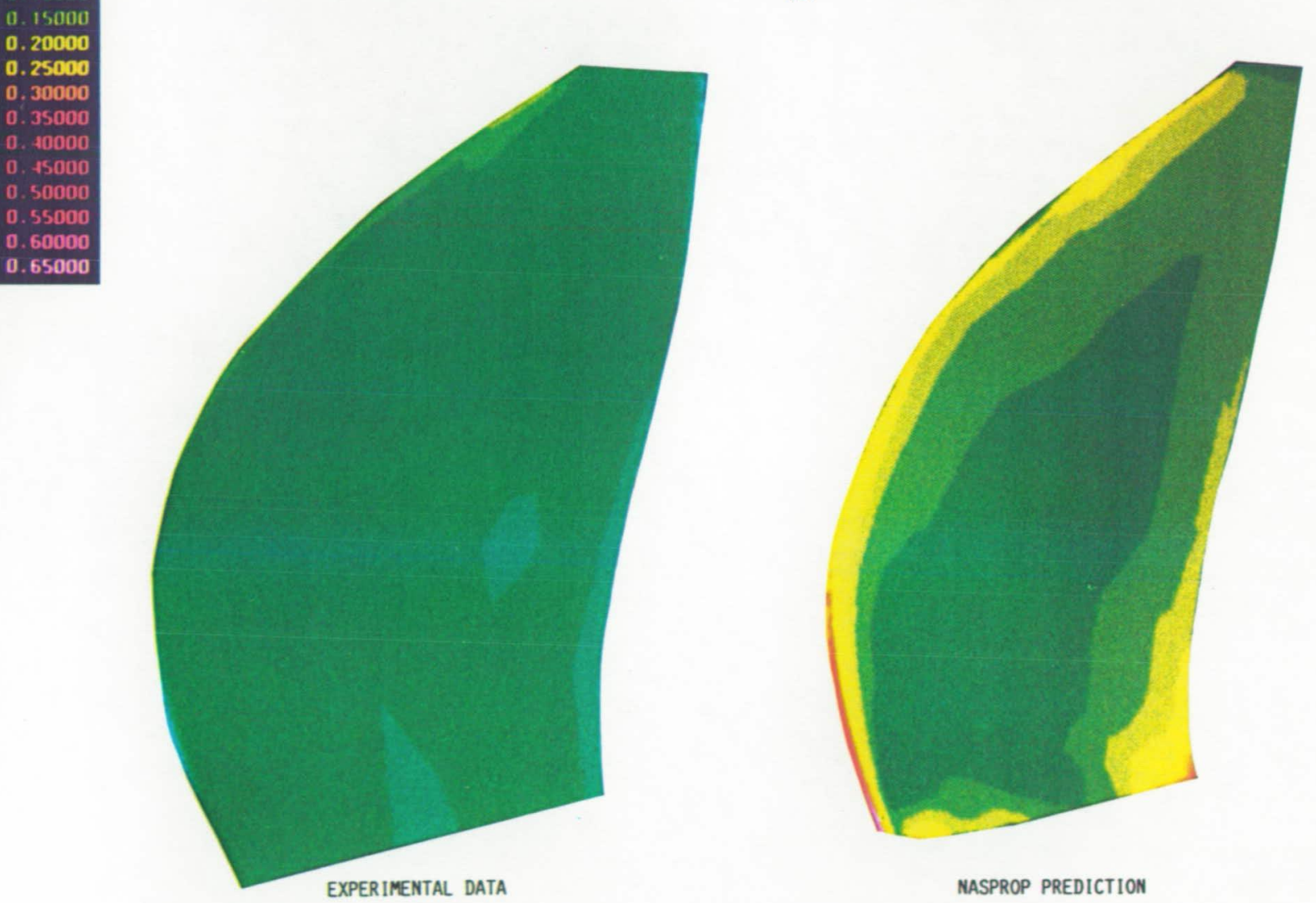

NASPROP PREDICTION

(b)

$$
\text { C-89-06144 }
$$

(a) Suction surface.

(b) Pressure surface.

Figure 6.-Comparison of predicted and measured pressure coefficient distributions for test 8 (Mach 0.5). 

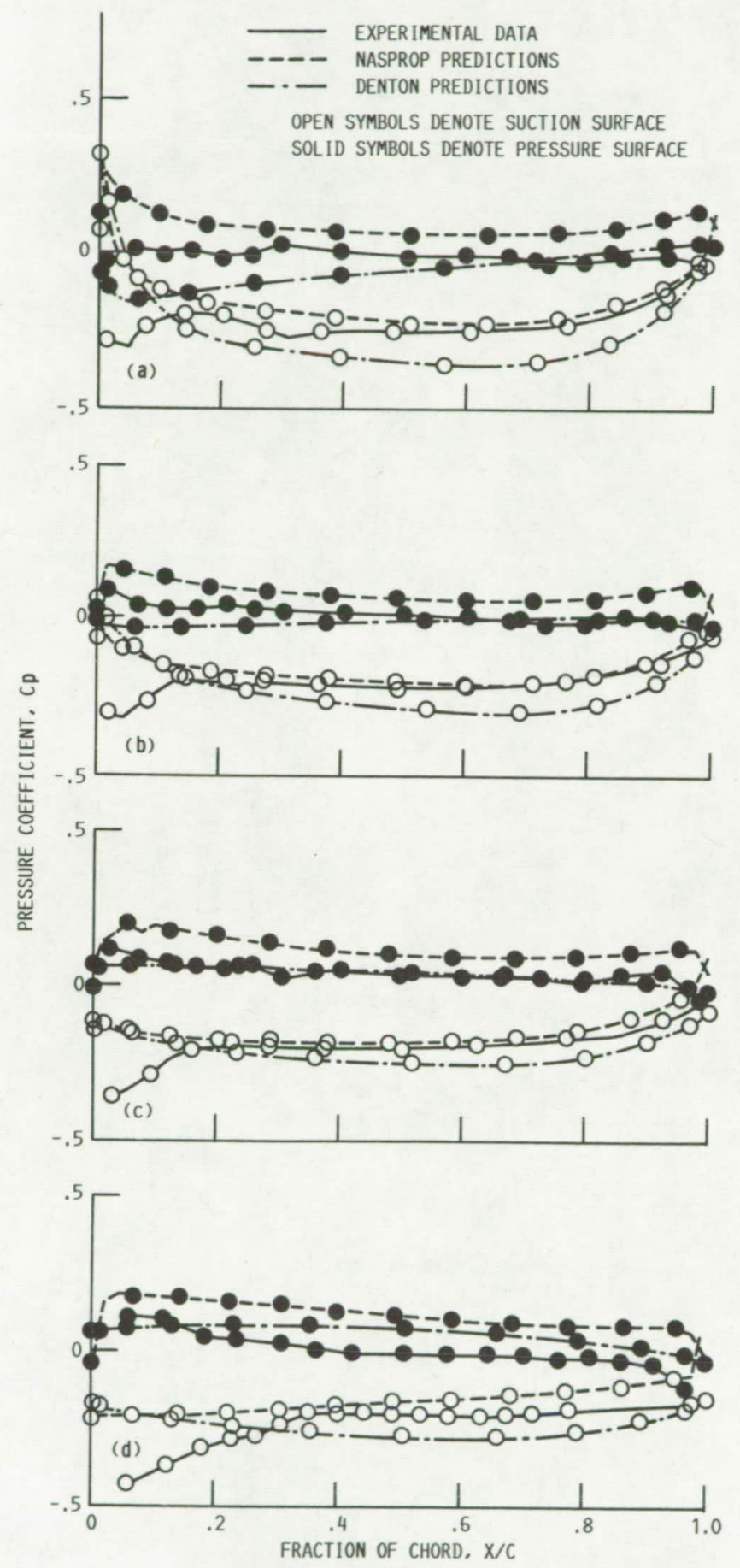

(a) Radial location, $r / R, 0.569$.

(b) Radial location, $r / R, 0.739$.

(c) Radial location, $r / R, 0.862$.

(d) Radial location, $r / R, 0.975$.

Figure 7.-Chordwise distribution of pressure coefficients for test 8 (Mach 5). 


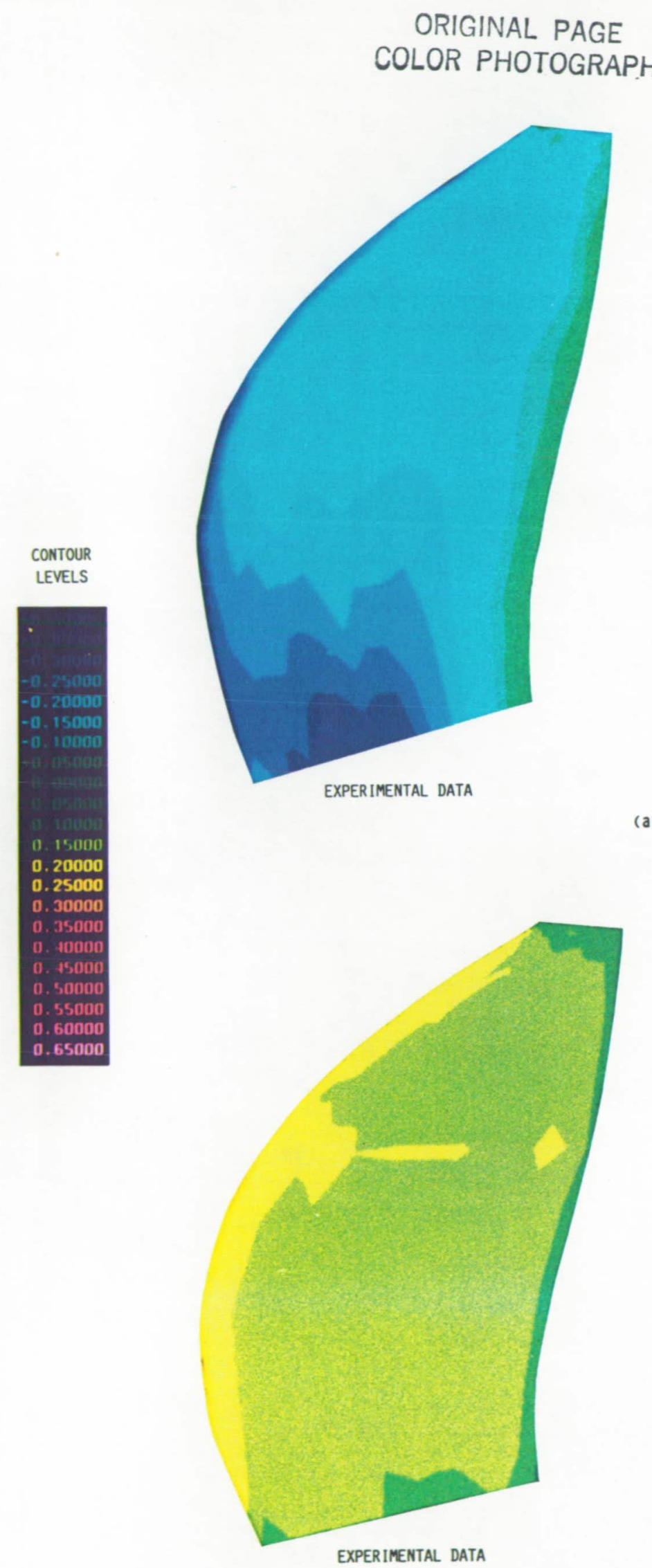

EXPERIMENTAL DATA

(a)
ORIGINAL PAGE IS OF POOR QUALITY

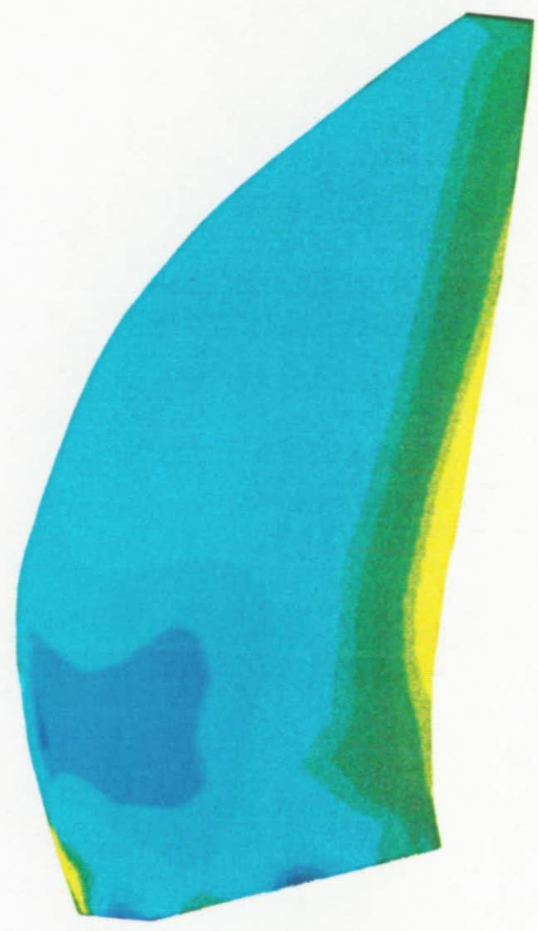

NASPROP PREDICTION

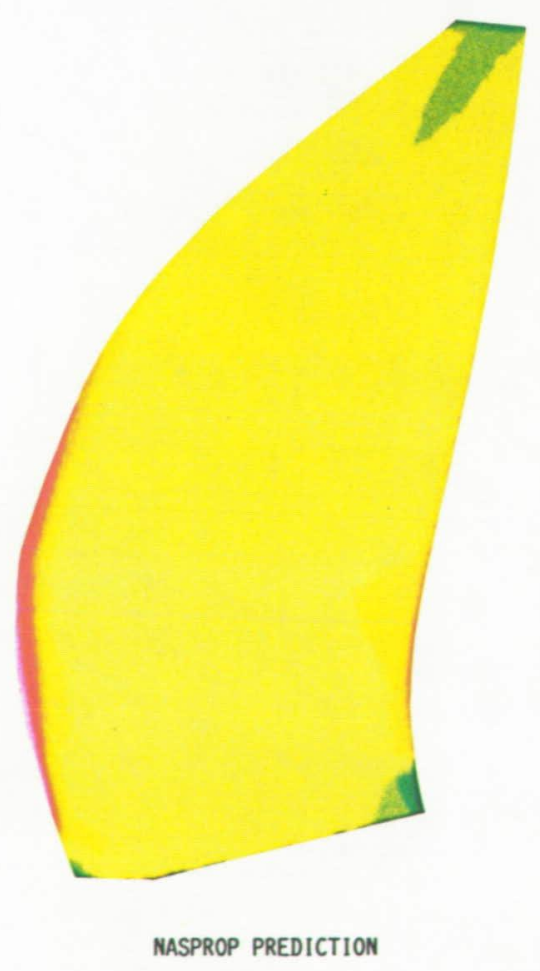

(b) C-89-06145

(a) Suction surface.

(b) Pressure surface.

Figure 8.-Comparison of predicted and measured pressure coefficient distributions for test 5 (Mach 0.2). 

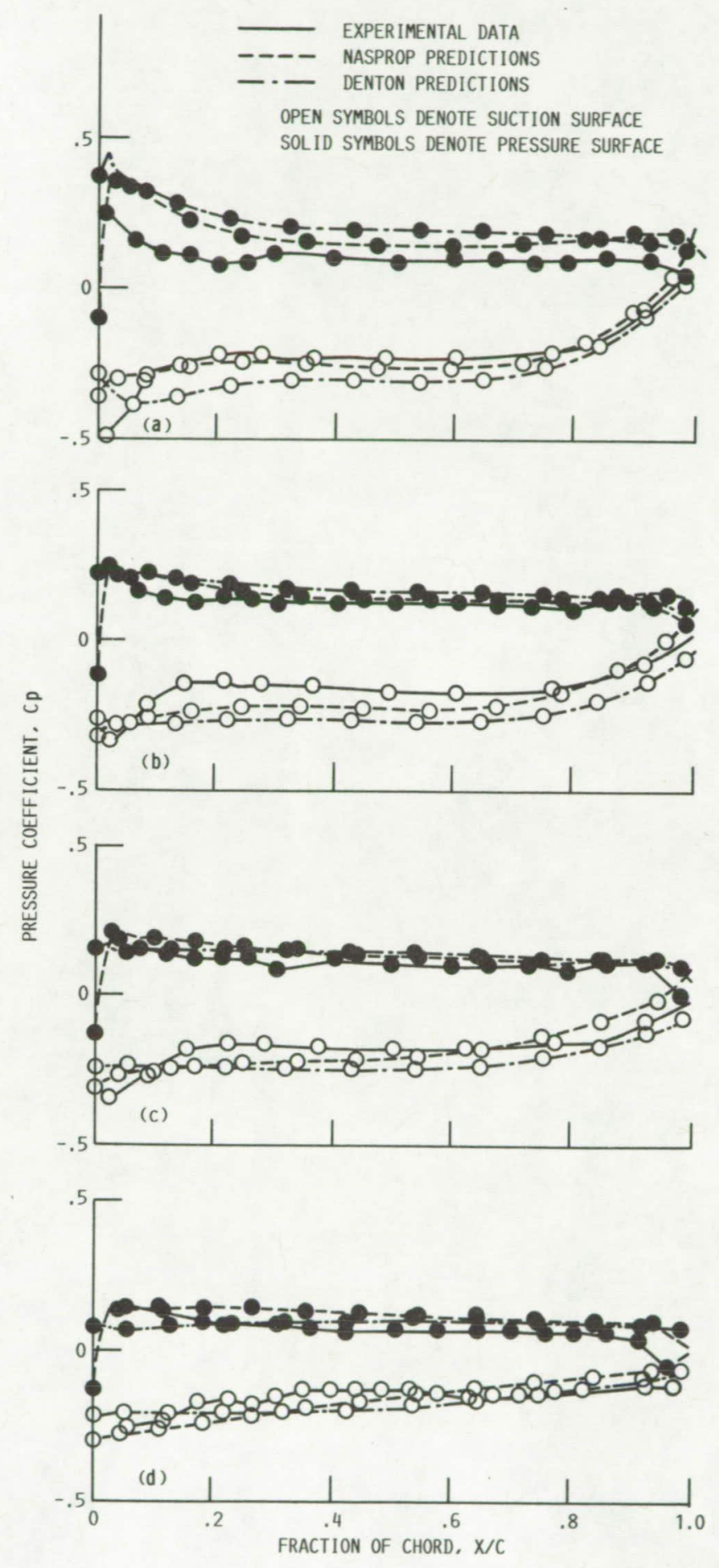

(a) Radial location, $r / R, 0.569$.

(b) Radial location, $r / R, 0.739$.

(c) Radial location, $r / R, 0.862$.

(d) Radial location, $r / R, 0.975$.

Figure 9.-Chordwise distribution of pressure coefficients for test 5 (Mach 0.2). 

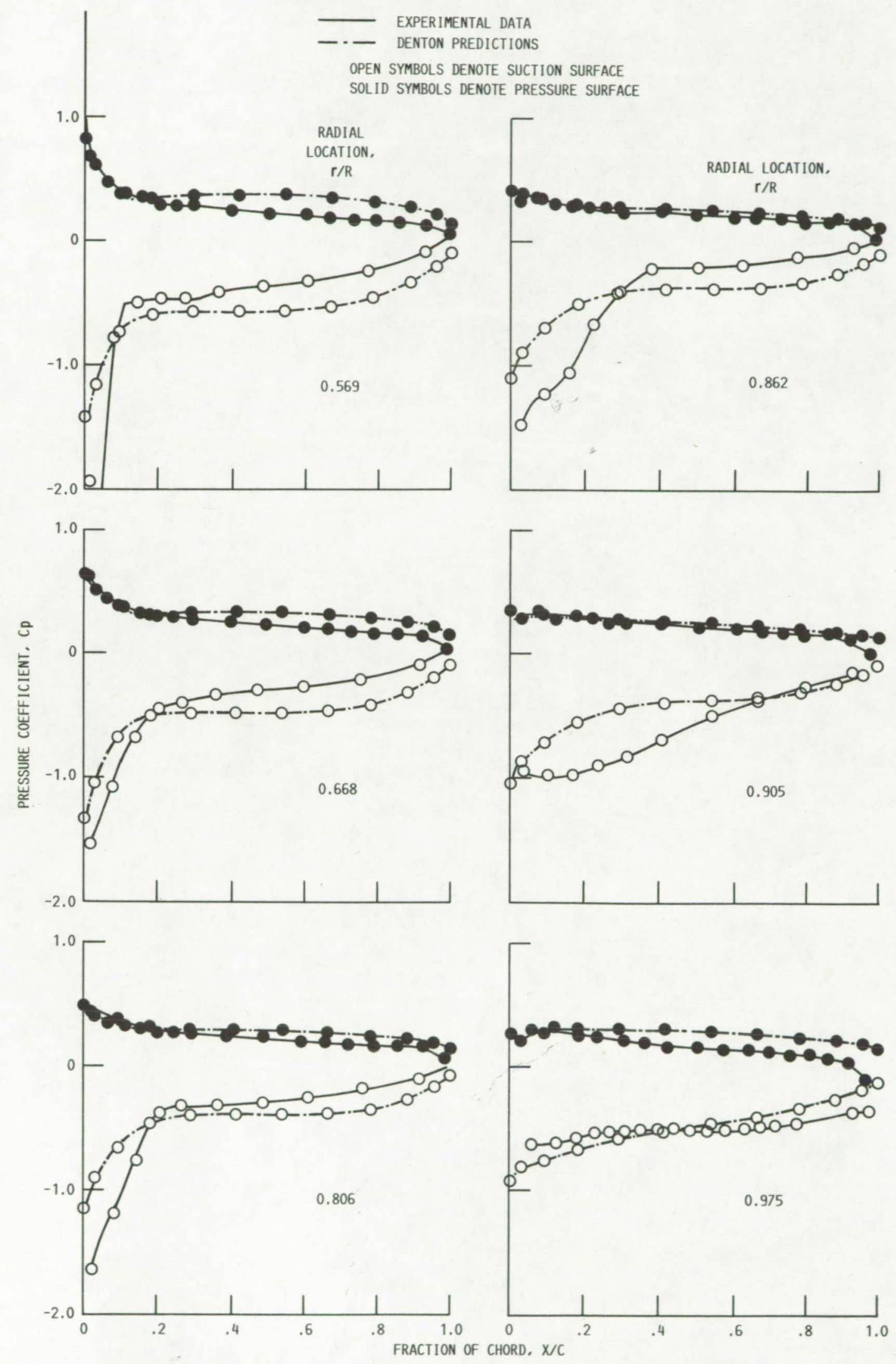

Figure 10.-Comparison of predicted and measured pressure coefficients for test 6 (Mach 0.2). 


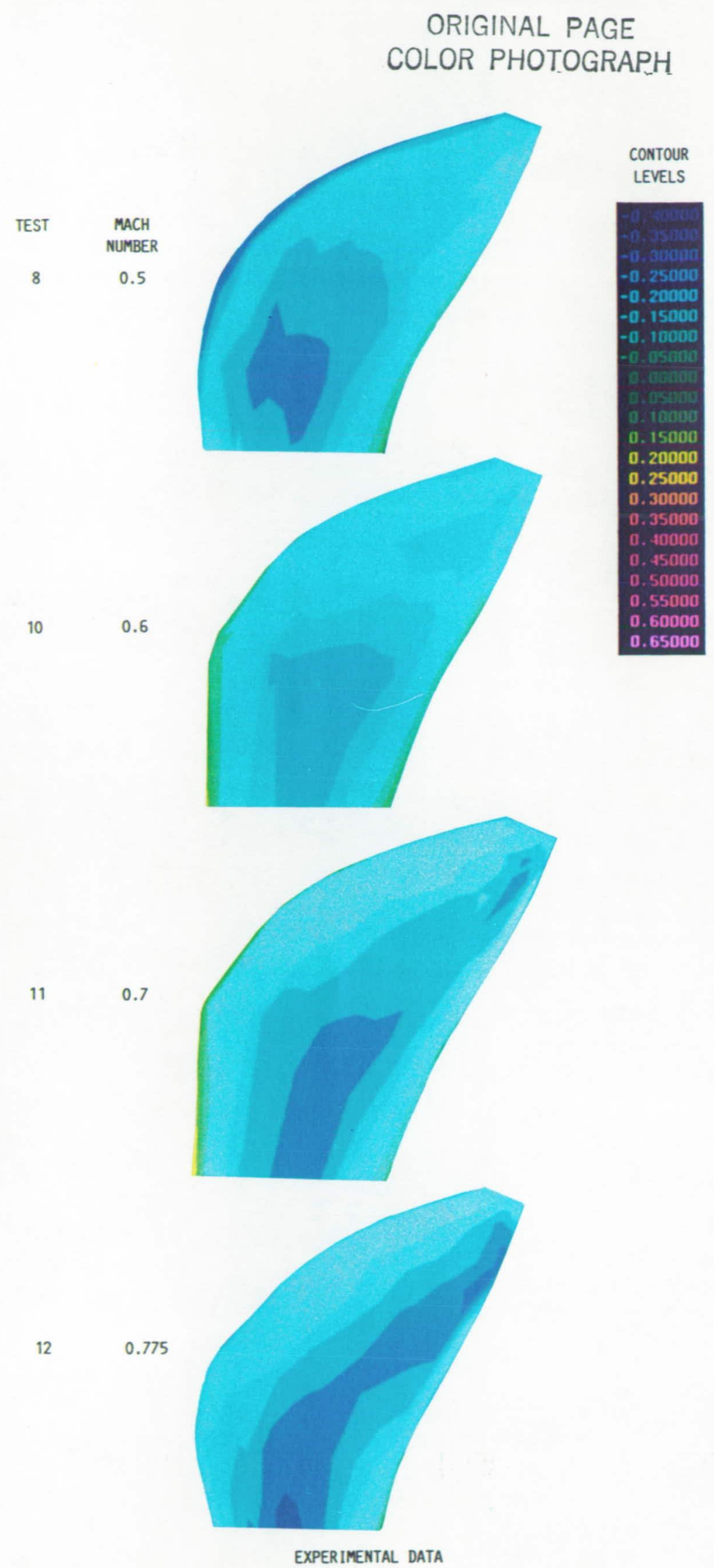

(a) Suction surface.
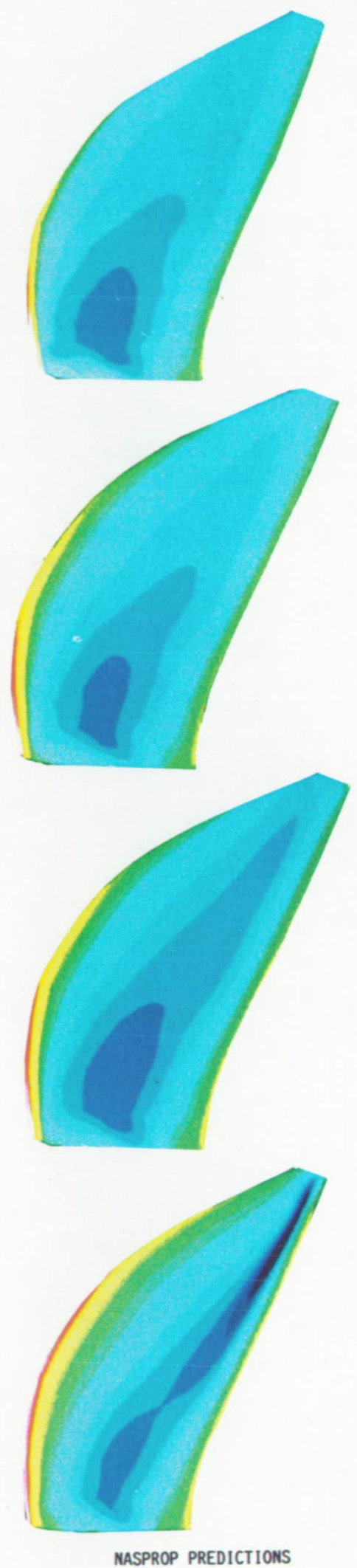

C-89-06146

Figure 11.-Comparison of predicted and measured compressibility effects: pressure coefficient distributions for various Mach numbers. 


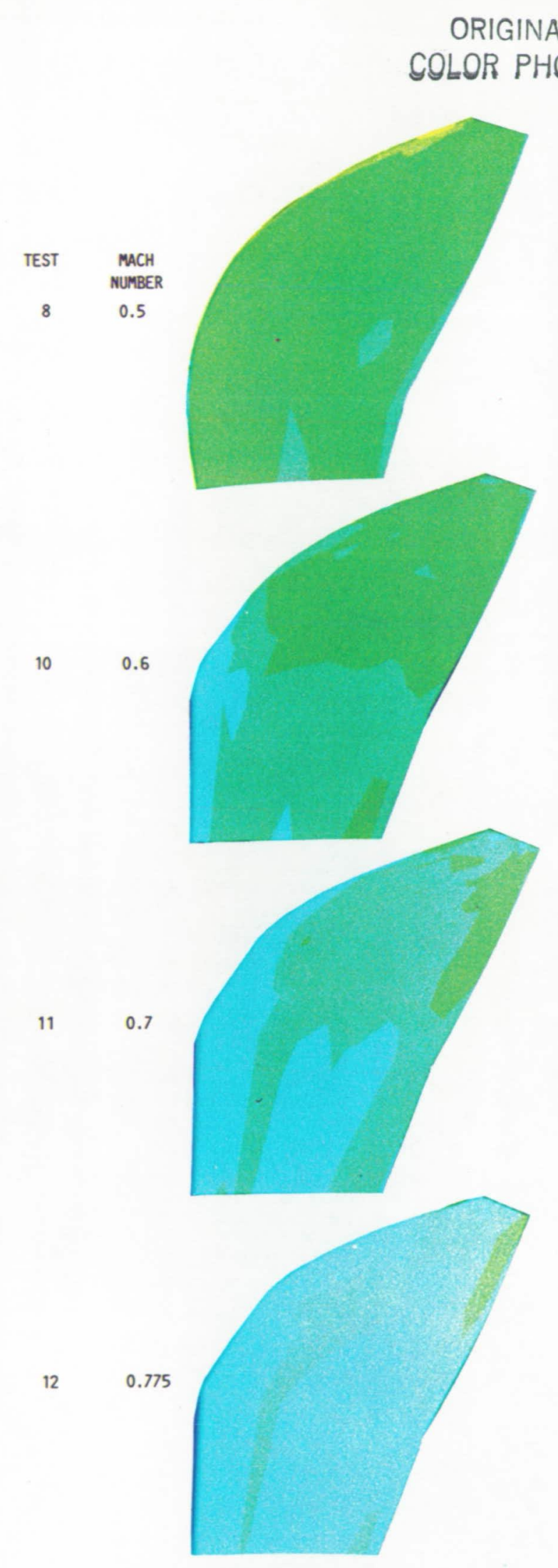

EXPERIMENTAL DATA
ORIGINAL PAGE IS OF POOR QUALITY

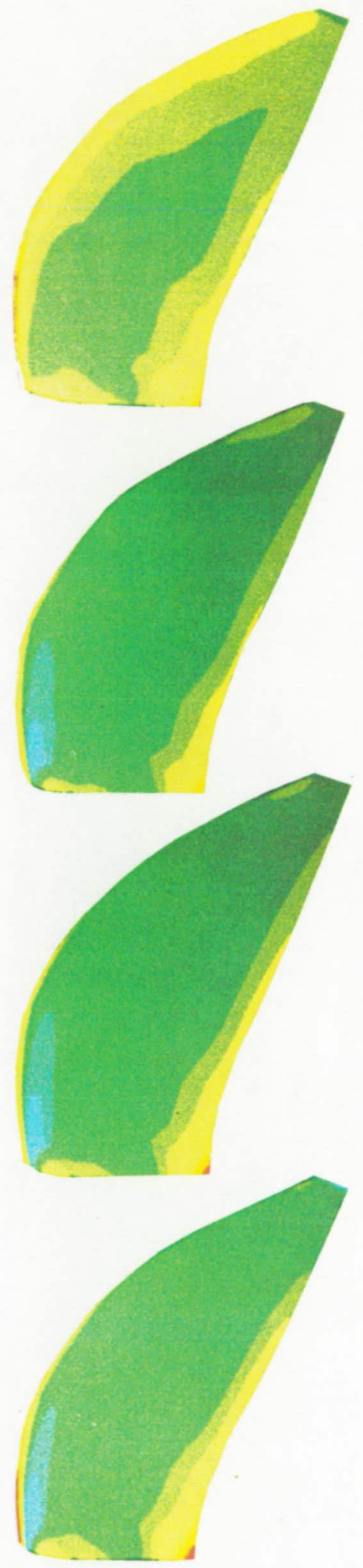

NASPROP PREDICTIONS

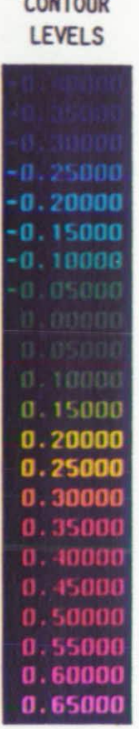

(b) Pressure surface.

C-89-06147

Figure 11.-Concluded. 


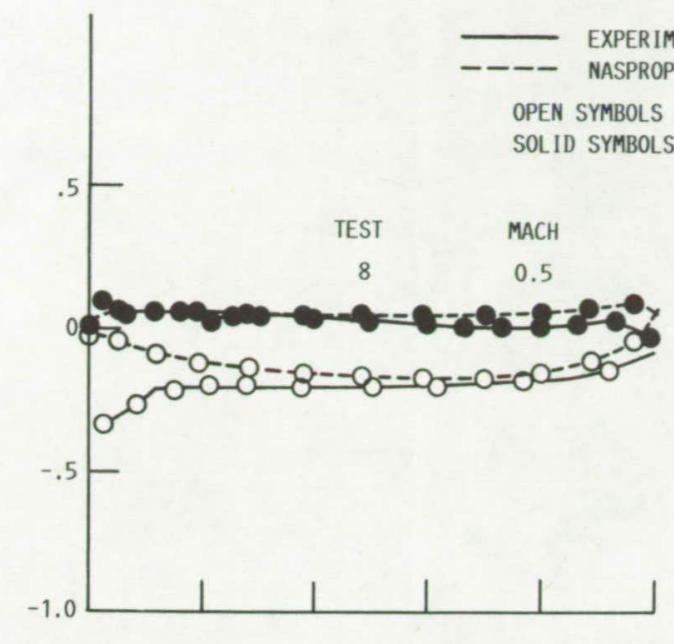

\section{EXPERIMENTAL DATA}

OPEN SYMBOLS DENOTE SUCTION SURFACE

SOLID SYMBOLS DENOTE PRESSURE SURFACE
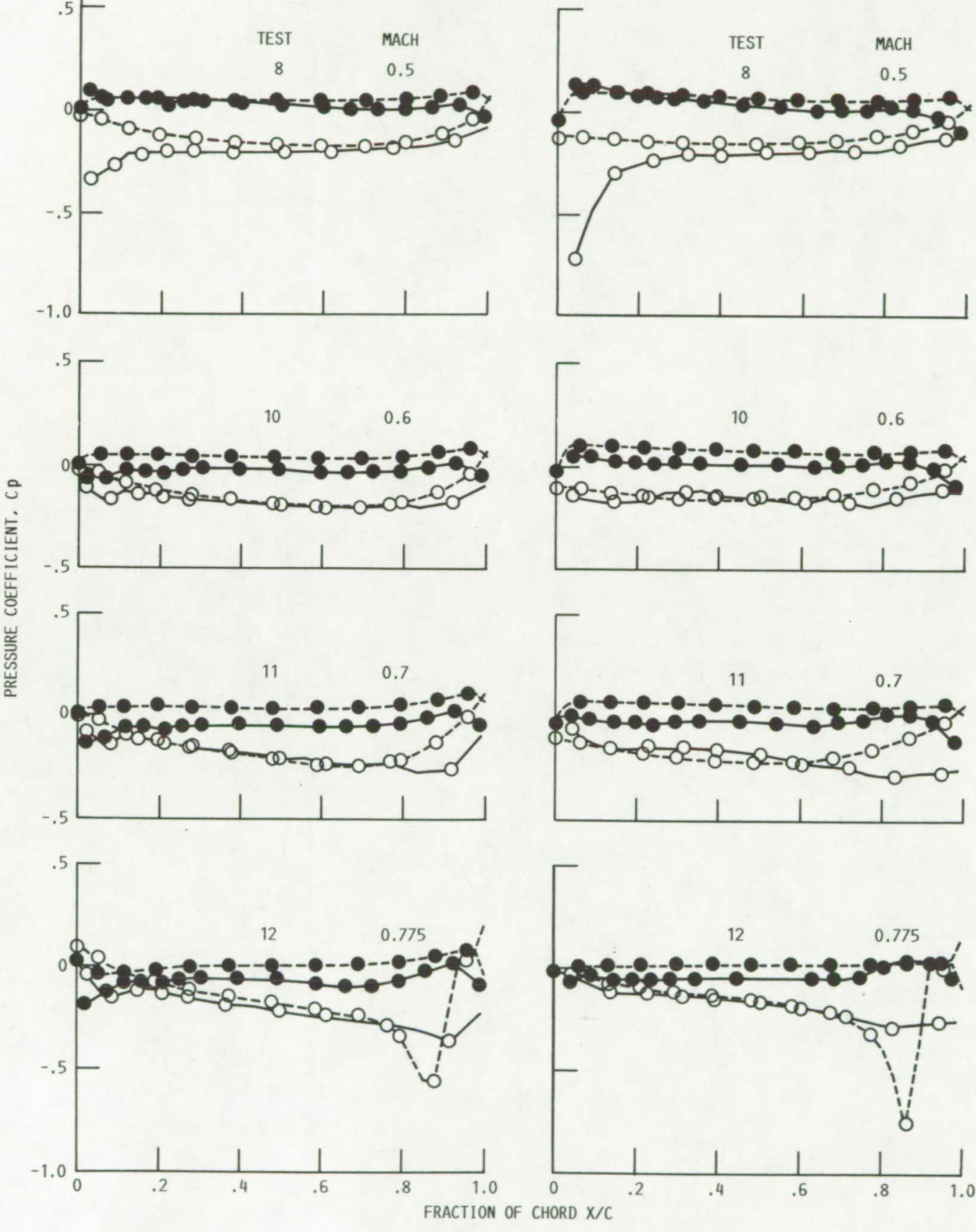

(a)

(b)

(a) Radial location, $r / R, 0.8060$.

(b) Radial location, $r / R, 0.9390$.

Figure 12.-Comparison of predicted and measured compressibility effects: chordwise distribution of pressure coefficients for two radial locations for various Mach numbers. 


\begin{tabular}{|c|c|c|}
\hline 1. Report No. & $\begin{array}{l}\text { NASA TM-102316 } \\
\text { AIAA-89-2696 }\end{array}$ & 2. Government Accession No. \\
\hline
\end{tabular}

4. Title and Subtitle

Large-Scale Advanced Propeller Blade Pressure Distributions:

Prediction and Data

7. Author(s)

M. Nallasamy, O. Yamamoto, S. Warsi, and L.J. Bober
3. Recipient's Catalog No.

5. Report Date

6. Performing Organization Code

8. Performing Organization Report No. E-4914

10. Work Unit No. 535-03-01

11. Contract or Grant No.

13. Type of Report and Period Covered Technical Memorandum

14. Sponsoring Agency Code

National Aeronautics and Space Administration

Washington, D.C. 20546-0001

15. Supplementary Notes

Prepared for the 25th Joint Propulsion Conference cosponsored by the AIAA, ASME, SAE, and ASEE, Monterey, California, July 10-12, 1989. M. Nallasamy, O. Yamamoto, and S. Warsi, Sverdrup Technology, Inc., NASA Lewis Research Center Group, Cleveland, Ohio 44135; L.J. Bober, NASA Lewis Research Center.

16. Abstract

Two Euler analysis techniques-finite difference and finite volume-were employed to predict the blade surface pressure distributions of a large-scale advanced propeller. The predicted pressure distributions were compared with wind tunnel data. Both techniques produced blade pressure distributions that are in fairly good agreement with the data over the range of test Mach numbers of 0.2 to 0.78 . However, the numerical simulations fail to predict correctly the measured pressure distributions for the low Mach number, high-power case. The data indicate the presence of a leading-edge vortex for this case. A discussion of the compressibility effects is also presented.

17. Key Words (Suggested by Author(s))

Propeller

Propfan

Blade pressure distributions

Euler analysis
19. Security Classif. (of this report)

Unclassified
20. Security Classif. (of this page)

Unclassified
18. Distribution Statement

Unclassified-Unlimited

Subject Category 07 